\title{
Software for muscle fibre type classification and analysis
}

\author{
P. Karen, ${ }^{1}$ M. Števanec, ${ }^{2}$ V. Smerdu, ${ }^{2}$ E. Cvetko, ${ }^{2}$ L. Kubínová, ${ }^{1}$ I. Eržen ${ }^{2}$ \\ ${ }^{1}$ Department of Biomathematics, Institute of Physiology, Academy of Sciences of the Czech Republic, \\ v.v.i., Prague, Czech Republic; ' Institute of Anatomy, Faculty of Medicine, University of Ljubljana, \\ Ljubljana, Slovenia
}

\begin{abstract}
(C)2009 European Journal of Histochemistry
Fibre type determination requires a large series of differently stained muscle sections. The manual identification of individual fibres through the series is tedious and time consuming. This paper presents a software that enables (i) adjusting the position of individual fibres through a series of differently stained sections (image registration) and identification of individual fibres through the series as well as (ii) muscle fibre classification and (iii) quantitative analysis. The data output of the system is the following: numerical and areal proportions of fibre types, fibre type size and optical density (grey level) of the final reaction product in every fibre. The muscle fibre type can be determined stepwise, based on one set of stained sections while further, newly stained sections can be added to the already defined muscle fibre profile. Several advantages of the presented software application in skeletal muscle research are presented. The system is semiquantitative, flexible, and user friendly.
\end{abstract}

Key words: skeletal muscle, muscle fibre types, mismatched fibres, myosin heavy chain isoforms, myosin heavy chain transcripts, oxidative and glycolytic fibres.

Correspondence: Ida Eržen,

Institute of Anatomy, Faculty of Medicine

University of Ljubljana, Korytkova 2, SI-1000 Ljubljana

Slovenia

Tel.: 00386.543.73.12.

Fax: 00386.543.73.01.

E-mail: ida.erzen@mf.uni-lj.si

Paper accepted on 26 May 2009

European Journal of Histochemistry

2009; vol. 53 issue 2 (April-June): 87-95
S keletal muscles are composed of muscle fibre types with different physiological and histochemical characteristics. Physiological, pathological and experimental conditions affect the proportion of fibre types, trigger fibre type transformations and affect fibre girth, whereby different fibre types could react in a different way. Area pertaining to muscle fibres that are stained with a particular procedure (e.g., demonstration of the activity of an enzyme, expression of a chosen antigen or mRNA transcript etc.) can quickly be estimated by the point counting method (e.g., Snoj-Cvetko et al., 1996a,b; Eržen et al., 1999; 2000). This method, however, provides no information on the fibre type pattern.

Fibre types can be determined from a successive series of differently stained cross sections. There exist different classification schemes, mostly based either on the lability of the myofibrillar adenosintriphosphatase (mATPase) in acid and alkaline media (Brooke and Kaiser, 1970) and according to the activity of the enzymes of energy supplying metabolism (Novikoff et al., 1961; Kugler, 1991; Reichmann and Pette, 1991), or the expression of myosin heavy chain isoforms (MyHC) (Schiaffino et al., 1989), etc. The classification of fibre types thus requires a large series of differently stained successive sections, and means a comparison of many images among each other, which is tedious and requires sustained attention of the human operator, possibly leading to errors. Therefore, computer assistance is desirable and software helping with identification of individual fibres and with assigning the histochemical profile is necessary to eliminate errors and speed up such analysis.

The first computer-assisted system for fibre type determination and quantitative analysis was developed by Pernuš et al. (1986). It required photos of successive sections stained for mATPase as well as enzymes indicating oxidative or glycolytic ways of energy supplying metabolism. The operator had to 
outline individual fibres on one photo only, laid on a digital tablet that was connected with a computer. Further analysis and classification was based on adjusting the xy coordinates of the fibre centroids that were assigned high, low or intermediate intensity of the reaction product, through a series of photos.

The programme was successfully applied with human (Pernuš and Eržen, 1991; Snoj-Cvetko and Eržen, 1992; Meznarič-Petruša et al., 1992; Pernuš and Eržen, 1994; Travnik et al., 1995; Čebašek et al., 1996; Eržen et al., 2000; Dahmane et al., 2001) and pig muscles (Škorjanc et al., 2007), proving itself to be a useful tool. However, it was necessary to manually lay the photos on the tablet and all judgements were left upon the decision and experience of the human operator. Therefore, the analysis was still quite tedious and sensitive to erroneous decisions of the operator. Further research aimed at an automatic segmentation of individual fibres on digital images. Different approaches were applied like Voronoi polygons, snakes, region growing, interfiber network skeletization, region and edge based active contours etc. (cf Lefaucher et al., 1992; Buche and Mauron, 1997; Klemenčič et al., 1998; Henckel et al., 1998; Brox et al., 2006; Dryden et al., 2006 and others). According to our experience none of the automatic methods provided satisfying segmentation on all kinds of stained muscle samples. A satisfying segmentation could be achieved with some muscle samples only, while in others a manual correction was necessary. Compared to the manual outlining on one image only, the automatic segmentation with the manual correction was much more time consuming and differed from sample to sample.

Therefore, a new, less tedious computer-assisted approach is necessary for more extensive morphometric studies employing the fibre type classification. Such system should make the identification of an individual fibre through a series of differently stained muscle sections more convenient, even in the case that not all muscle fibres run parallel to each other within a muscle sample and that fibres change their shape through a large series of sections, and if some fibres disappear from the section or they split as well. Further, the new computer-assisted approach should take into account that through a large series of reactions artefacts may appear in different places of serial sections.
Moreover, as the specificity of the antibodies against MyHC isoforms may vary among the two species, the system should provide the ability to adjust the fibre type identification in rat and in human muscles (Smerdu and Soukup, 2008).

In this paper we present such a computer-assisted system applying a new approach with manual delineation of fibre outlines in one muscle section and semi-automatic registration of successive muscle sections, which enables the fibre type classification. The software implementation is described and its advantages for skeletal muscle research are demonstrated on the study of test samples of human and rat skeletal muscles.

\section{Materials and Methods}

\section{Muscle samples}

To test the new methodology, the samples of human biceps femoris, vastus lateralis and masseter muscles, taken at autopsy or biopsy and rat extensor digitorum longus, tibialis anterior and gastrocnemius muscles were excised, deeply frozen in liquid nitrogen and cut into $10 \mu \mathrm{m}$ thick transverse sections. The sections were processed to demonstrate the expression of MyHC isoforms and key enzymes of energy supplying metabolism, i.e. NADH-tetrazolium reductase (NADH-dehydrogenase, Novikoff et al., 1961), succinate dehydrogenase (SDH, Reichmann and Pette, 1991), glycerol-3-phosphate dehydrogenase $(\alpha-G P D H$, Kugler, 1991).

\section{Antibodies}

To reveal the $\mathrm{MyHC}$ isoforms the following antibodies were applied: BA-D5 (MyHC-1), SC-71 (MyHC-2a in rat and MyHC-2a and $2 x$ in humans), BF-35 (against all MyHC isoforms except $-2 x$ ) and BF-F3 (MyHC-2b). These antibodies are specific to rat $\mathrm{MyHC}$ isoforms (Schiaffino et al., 1989). Additionally, A4-74 that is according to the manufacturer (Alexis Biochemicals) specific to MyHC-2a of human, mouse, rat and rabbit, F113.15F4, specific to MyHC-2a and $-2 b$ of various species; and $6 \mathrm{HI}$, specific to MyHC-2x of various species (Lucas et al., 2000) were used as well. The corresponding cell line was gifted by Dr. L. Leinwand (Department of MCD Biology, University of 
Colorado, Boulder, USA), the supernatant was produced in the local laboratory. The protocols used for immunohistochemistry are described in Smerdu and Soukup (2008).

\section{In situ hybridisation}

To reveal the MyHC transcripts (mRNAs) expression in the fibres of human biceps femoris muscle, serial cryosections were processed for in situ hybridisation with probes, specific to MyHC transcripts of $\beta$-slow, $2 \mathrm{a}$ and $2 \mathrm{x}$ MyHC genes (Smerdu et al., 1994; Weiss et al., 1999). The riboprobes were prepared with digoxigeninlabelled UTP according to the guidelines of the manufacturer (Roche Molecular Biochemicals). The procedure was adapted from Horton et al. (2001), with the following modifications: the sections were digested with $20 \mu \mathrm{g} / \mathrm{mL}$ of proteinase $\mathrm{K}$ for 7.5 minutes and the site of probe hybridisation was revealed with sheep anti-digoxigenin Fabalkaline phosphatase conjugate (1:2000).

\section{Image acquisition}

The images of muscle sections were captured from differently stained serial sections by the Nikon digital camera DXM1200F connected to the Nikon Eclipse E800 microscope applying the Lucia GF software (version 4.82).

\section{Software description}

Two consecutive tasks leading to the muscle fibre classification, i.e. (i) the registration of serial physical muscle sections and (ii) the actual classification of fibre types, were implemented in two partially independent programmes in $\mathrm{C}^{++}$programming language, running on computers with Microsoft Windows operating system. Both programmes were developed using a "wizard-style", i.e. the successive panels of the main dialog window were arranged in a sequence of individual steps leading the user to proceed in a natural way during the execution of the task. However, the system allows returning to previous stage at any step, retaining all the work that has been already done. It also enables to save a current status of the task into a special file, enabling to return to any stage of work later, by loading it back again. An instant visual feedback provides the user comprehensive information and the insight in the process of fibre type classification during the run of both programmes.

\section{MuscRegM programme}

A set of images acquired from differently stained successive physical muscle sections represents the input data to the programme. The user determines and marks several mutually corresponding control points within the images. The number of points needed for the desired precision of the registration is influenced by the degree of distortion of the source images. Typically, from 4 to 8 points are sufficient in most real-world cases (Figure 1a).

The user should appoint one single image as the reference/master one. An elastic registration algorithm (Bookstein, 1989; Likar and Pernuš 1999; Čapek et al., 2009) is then applied to warp the rest of images to line-up corresponding control points. The resulting set of the registered images is finally saved for further usage (Figure $1 a$ ).

\section{Intermediate step: contours of individual muscle fibres}

The commercial image analysis programme Ellipse (ViDiTo, Košice, Slovakia) is used for convenient outlining contours of individual muscle fibres within the reference/master image only (see the description of MuscRegM programme).

\section{FibClasM programme}

A set of registered images obtained as the result of previous MuscRegM programme represented the input data to the programme. Then the contours of individual muscle fibres corresponding to the reference/master image were loaded and superimposed on this image. These contours were exploited for identification of the relevant contours in the images of other serial muscle sections. Taking into account that the image registration is never perfect and it is not necessary to employ the whole fibre area for classification, the fibre contours can be slightly scaled down before copying them into the non-master images. This ensures that the position of contours is acceptable in the most cases even if the images are not well registered, while the smaller area still remains sufficient for the fibre type classification. The user can choose the "shrinkage ratio" according to his needs (the better the registration, the lower the "shrinkage ratio" can be applied). If the above method of the transferring fibre contours fails to meet the user's ideas, it is possible to move and/or resize the individual con- 


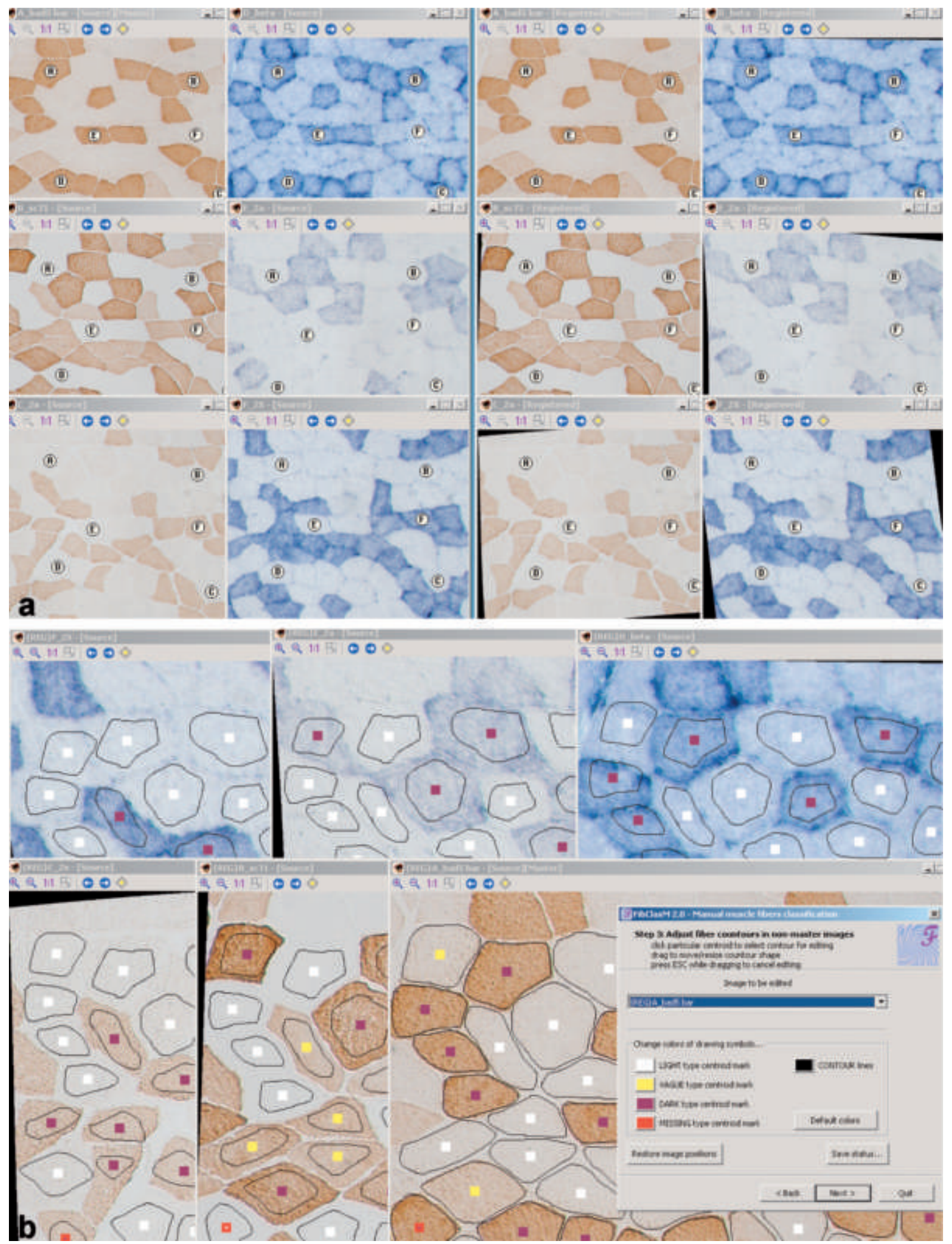

Figure 1. Muscle fibre registration applying MuscRegM programme (Figure 1a) and muscle fibre type classification applying FibReg programme (Figure 1b) in serial sections of human biceps femoris muscle. In Figure 1a the original images are arranged in the first two columns and the registered images in the third and fourth column. The first image of the first column and the first image of the third column are 'the master' images (the first column presents immunohistochemical demonstration of myosin heavy chain isoforms MyHC-1, MyHC-2a, MyHC-2x whilst the second column presents the corresponding mRNA transcripts for MyHC-1, MyHC-2a, MyHC-2x). Figure 1b shows the thresholding of the registered images, which is the essential step in the fibre classification. 
tour within a particular image manually.

The mean luminance of the area outlined by the fibre contour was employed for the thresholds setting and the subsequent classification of the muscle fibre types. Two thresholds can be interactively set by the user for each image to define a dark, intermediate and light feature of individual fibres. The initial values of both thresholds are computed according to the Otsu method (Otsu, 1979; Sezgin and Sankur, 2004) to obtain an "optimal" clustering of dark and light fibres. This method is based on minimizing the weighted within-cluster variance that turns out to be the same as maximizing the between-cluster variance.

If the automatic classification based on thresholds is not acceptable for some particular fibre, the user can manually determine the type of individual fibre. It is also possible to mark the fibre as a missing one, if the classification is infeasible (Figure 1b).

An exhaustive outline of the resulting classification in the form of MS Excel file can be created and saved. The file consists of several sheets reflecting various perspectives of the result data. Images sheet yields the characteristics of individual images, Fibres sheet displays the characteristics of all individual fibres, Profiles sheet shows the characteristics of all profiles (i.e. the mutual fibre density classifications) found. The rest of sheets were named as Compound profiles and they yield the characteristics of individual fibres falling into the particular profile that reflects muscle fibre type. Among others, the individual or mean values of various geometrical characteristics (area, perimeter, Feret's diameters, etc.) and optical density (grey level) are computed and listed for individual fibres and fibre types, respectively.

\section{Further data processing}

For the experiment specific purposes several macros have been developed that operate on Excel result file and can be used to extract and/or transform data according to particular needs. For example, based on different specificity of the applied antibodies to $\mathrm{MyHC}$ isoforms in rat and human muscles (Smerdu and Soukup, 2008), the fibre types are determined by selecting staining combinations through a set of images (0-dark, 1intermediate and 2-light), for rat and human muscles separately.

\section{Results}

The described programme provides the data about fibre types and their morphometric characteristics, e.g., diameter, perimeter, cross-sectional area, and their percentage within a sample as well as the grey level in every fibre through the series of variously stained sections. The fibre type classification based on different features, e.g., the expression of MyHC isoforms (Table 1), MyHC transcripts expression within muscle fibres and the activity of energy supplying enzymes. Additionally, it is possible to add other characteristics obtained with other specific staining reactions (e.g., the expression of different proteins, important in the diagnostics of the neuromuscular diseases) (Table 2) to fibre

Table 1. Data output from fibre type analysis in rat tibialis anterior muscle. Successive serial sections were stained for myosin heavy chain (MyHC) isoforms MyHC-1, MyHC-2a, MyHC-2x and MyHC-2b.

\begin{tabular}{lccc}
\hline Fibre type & $N$ & $\%$ & Diameter $(\mu m)$ \\
\hline 1 & 2 & 1.5 & 24.0 \\
$2 a$ & 24 & 17.6 & 23.2 \\
$2 \mathrm{x}$ & 34 & 25.0 & 32.9 \\
$2 \mathrm{~b}$ & 33 & 24.3 & 45.5 \\
$1 / 2 \mathrm{a}$ & 0 & 0.0 & - \\
$1 / 2 \mathrm{a} / 2 \mathrm{x}$ & 0 & 0.0 & - \\
$2 \mathrm{a} / 2 \mathrm{~b}$ & 1 & 0.7 & 27.0 \\
$2 \mathrm{a} / 2 \mathrm{x} / 2 \mathrm{~b}$ & 0 & 0.0 & - \\
$1 / 2 \mathrm{x}$ & 2 & 1.5 & 23.5 \\
$1 / 2 \mathrm{a} / 2 \mathrm{x} / 2 \mathrm{~b}$ & 0 & 0.0 & - \\
$1 / 2 \mathrm{x} / 2 \mathrm{~b}$ & 0 & 0.0 & - \\
$1 / 2 \mathrm{a} / 2 \mathrm{~b}$ & 0 & 0.0 & - \\
$1 / 2 \mathrm{~b}$ & 0 & 0.0 & - \\
$2 \mathrm{~b} / 2 \mathrm{x}$ & 26 & 19.1 & 38.9 \\
$2 \mathrm{a} / 2 \mathrm{x}$ & 10 & 7.4 & 25.2 \\
$\mathrm{ncf}$ & 4 & 2.9 & 27.3 \\
\hline
\end{tabular}

ncf: nonclassified fibre.

Table 2. An example of adding a new information about fibre type features to already classified fibres in human masseter muscle. The muscle fibres were classified according to the expression of MyHC-1, MyHC-2a and MyHC-2x. Additional feature is the expression of neonatal myosin. Staining level: 0dark, 1-intermediate, 2-light.

\begin{tabular}{lccccc}
\hline & & & \multicolumn{3}{c}{ Additional feature staining level } \\
& & & 0 & 1 & 2 \\
\hline Fibre type & $N$ & $(\%)$ & $N$ & $N$ & $N$ \\
\hline 1 & 31 & 30.39 & 3 & 3 & 25 \\
$2 \mathrm{a}$ & 13 & 12.75 & 0 & 0 & 13 \\
$2 \mathrm{x}$ & 2 & 1.96 & 0 & 0 & 2 \\
$2 \mathrm{a} 2 \mathrm{x}$ & 43 & 42.16 & 0 & 0 & 43 \\
$1 / 2 \mathrm{a}$ & 7 & 6.86 & 0 & 1 & 6 \\
$1 / 2 \mathrm{x}$ & 0 & 0.00 & 0 & 0 & 0 \\
$1 / 2 \mathrm{a} / 2 \mathrm{x}$ & 6 & 5.88 & 0 & 0 & 6 \\
\hline & & & & & \\
Sum & 102 & 100.00 & 3 & 4 & 95 \\
\hline
\end{tabular}


types already defined from a chosen set of reactions. The system is very helpful in the simultaneous analysis of the expression of $\mathrm{MyHC}$ isoforms and their mRNA transcripts (Table 3 ). The programme is efficient in the recognition of individual fibres through a set of images. Every fibre is numbered and its fibre type can be identified by the combination of various staining reactions through the series (profile) (Figure 2). Based on the specificity of antibodies to particular MyHC isoforms, the system provides the number of fibres assigned to a defined fibre type. For different reasons some fibres can not be assigned to any of the fibre

Table 3. Mismatch between the expression of MyHC isoforms and MyHC transcripts in one sample of human biceps femoris muscle.

\begin{tabular}{lcccccc}
\hline & \multicolumn{2}{c}{ MyHC transcripts } & \multicolumn{2}{c}{ MyHC } & \multicolumn{2}{c}{$\begin{array}{c}\text { Mismatch } \\
\text { MyHC transcripts/MyHC }\end{array}$} \\
\hline Fibre type & $N$ & $(\%)$ & $N$ & $(\%)$ & $N$ & $(\%)$ \\
\hline 1 & 19 & 38.85 & 20 & 37.74 & 1 & 8.33 \\
$2 \mathrm{a}$ & 11 & 20.75 & 7 & 13.21 & 4 & 33.33 \\
$2 \mathrm{x}$ & 21 & 39.62 & 1 & 1.89 & 5 & 41.67 \\
$2 \mathrm{a} 2 \mathrm{x}$ & 0 & 0.00 & 20 & 37.74 & 0 & 0.00 \\
$1 / 2 \mathrm{a}$ & 0 & 0.00 & 4 & 7.55 & 0 & 0.00 \\
$1 / 2 \mathrm{x}$ & 0 & 0.00 & 1 & 1.89 & 0 & 0.00 \\
$1 / 2 \mathrm{a} / 2 \mathrm{x}$ & 0 & 0.00 & 0 & 0.00 & 0 & 0.00 \\
ncf & 2 & 3.77 & 0 & 0.00 & 2 & 16.67 \\
\hline Sum & 53 & 100.00 & 53 & 100.00 & 12 & 100.00 \\
\hline
\end{tabular}

The fibre types in the left part of the table are defined according to the expression of MyHC transcripts. The fibre types in the middle part of the table are defined according to the expression of MyHC isoforms. In the right part of the table the absolute number (N) and percentage (\%) of fibres (within each fibre type) in which MyHC transcripts expression did not match to the expressed MyHC isoforms is presented.

Table 4. Efficiency of the classification procedure judged by the share of non-classified fibres in human masseter and vastus lateralis muscle.

\begin{tabular}{lcccccc}
\hline Sample & \multicolumn{3}{c}{ Masseter } & & \multicolumn{3}{c}{ Vastus lateralis } \\
& $N_{\text {tot }}$ & $N_{\text {ncf }}$ & ncf (\%) & $N_{\text {tot }}$ & $N_{\text {ncf }}$ & ncf (\%) \\
\hline 1 & 102 & 0 & 0.00 & - & - & - \\
2 & 93 & 7 & 7.53 & 82 & 6 & 7.32 \\
3 & 68 & 2 & 2.94 & 124 & 1 & 0.81 \\
4 & 129 & 0 & 0.00 & 111 & 1 & 0.90 \\
5 & 103 & 7 & 6.80 & 114 & 0 & 0.00 \\
6 & 98 & 1 & 1.02 & 99 & 2 & 2.02 \\
7 & 81 & 1 & 1.23 & - & - & - \\
8 & 105 & 3 & 2.85 & 102 & 0 & 0.00 \\
9 & 95 & 4 & 4.21 & 129 & 0 & 0.00 \\
10 & 103 & 6 & 5.83 & 82 & 1 & 1.22 \\
11 & 136 & 0 & 0.00 & 108 & 0 & 0.00 \\
12 & 104 & 1 & 0.96 & 108 & 0 & 0.00 \\
13 & 160 & 3 & 1.88 & - & - & - \\
14 & 115 & 4 & 3.48 & 153 & 2 & 1.31 \\
15 & 90 & 0 & 0.00 & 124 & 2 & 1.61 \\
\hline sum & 1582 & 39 & & 1336 & 15 & \\
average & & & 2.58 & & & 1.27 \\
\hline
\end{tabular}

$N_{\text {tot }}$ : the total number of fibres within a sample, $N_{\text {ncf }}$ the number of the non-classified fibres, ncf (\%) - the percentage of the non-classified fibres within a sample. groups. One reason is that these fibres could not be identified in one or more sections. These fibres are called non-classified fibres (ncf). The share of such non-classified fibres points to the efficiency of the classification procedure. Table 4 shows the share of ncf obtained in the analysis of fibres in human masseter and in vastus lateralis muscle. In the masseter muscle the identification of individual fibres through a set of images was quite demanding because muscle fibres are not parallel to each other but rather intermingled in the sample. In vastus lateralis muscle the analysis was much easier.

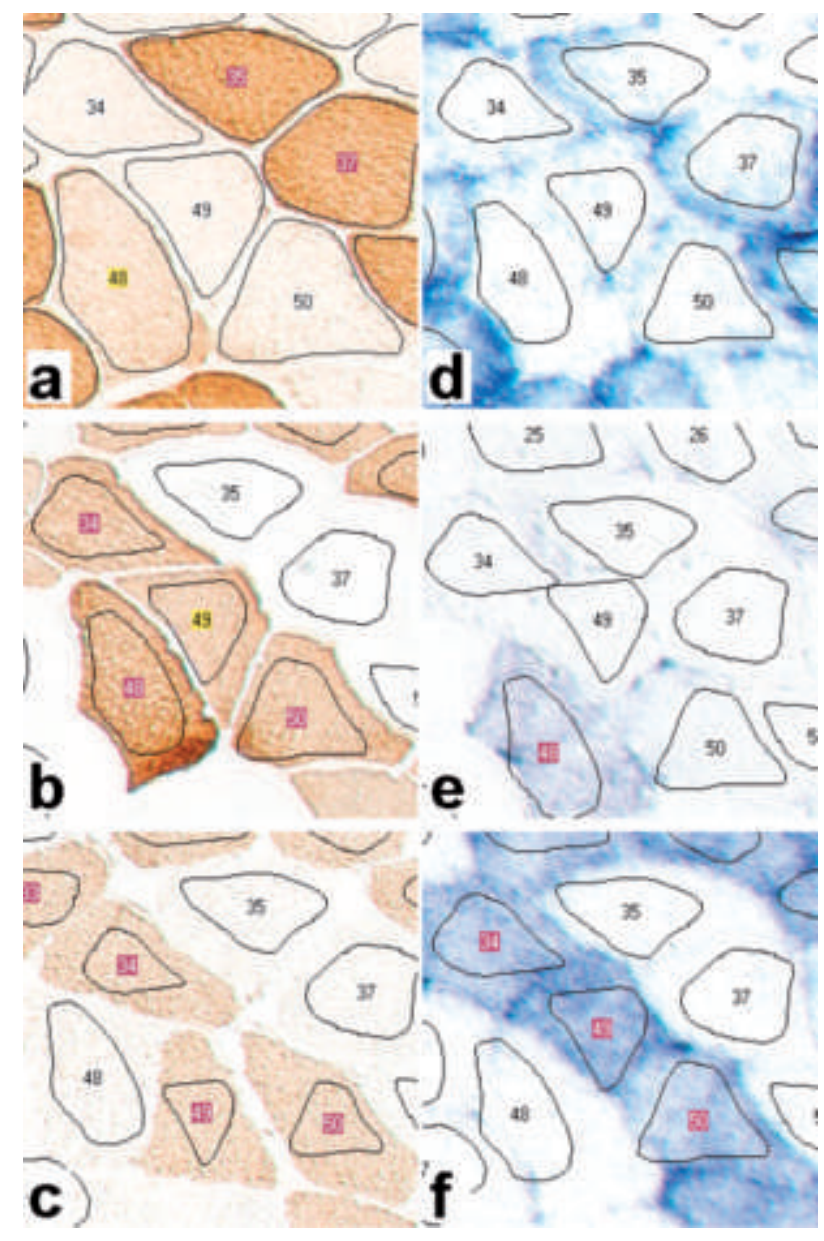

Figure 2. Mismatch between the MyHC isoforms and mRNA transcripts expression in human biceps femoris muscle. Immunohistochemical demonstration of MyHC-1 by BA-D5 (a), MyHC-2a (intensively) and -2x (moderately) by SC-71 (b), MyHC$2 x$ by $6 \mathrm{H} 1$ (c) isoforms and $\beta$-slow (d), 2a (e), 2x (f) MyHC transcripts expression revealed by in situ hybridisation technique. Note the mismatched fibre No. 48, expressing MyHC-1 (a) and 2a (b), thus classified as 1/2a fibre but expressing 2a MyHC transcripts only. Another mismatched fibre is No. 35, histochemicaly classified as type 1 , but hardly expressing corresponding $\beta$ slow MyHC transcripts. 


\section{Discussion}

This paper presents software for fibre type classification and the advantages of its application in skeletal muscle research.

The described classification and analysis of muscle fibre types requires outlining individual fibres in one image only. If automatic segmentation were feasible the whole analysis would be much quicker. Different methods for segmentation of muscle fibres (e.g., snakes, region growing. etc) have been tested, however, they are not reproducible and provide satisfying results in some selected samples only. In the majority of samples a manual correction is needed. Consequently, the whole procedure is more time consuming than manual segmentation itself.

Fibre registration through a successive series of differently stained muscle sections (MuscRegM programme) is especially helpful in samples where muscle fibres are not oriented parallel to each other. Such orientation is the reason why individual fibres change their shape and the $x y$ position of their centroids in some sections within a set of reactions in serial sections. Additionally, the programme helps with revealing splitted and missing fibres.

The grey level of a particular staining within individual muscle fibres that is set with thresholding to dark (0), intermediate ( 1 ) and light (2) respectively, is the basis of fibre classification.

However, for several reasons (the microscope settings, camera characteristics, uneven section thickness and other artefacts due to the tissue preparation and staining procedures) identical grey level in different portions of the image does not necessary mean the same fibre type. To avoid possible misclassification in such particular cases fibre label $(0,1,2)$ can be changed manually.

The system provides also the number of nonclassified fibres. These fibres are those that change their shape or they are missing, because the tissue section is torn or wrinkled or due to other local artefacts. Consequently, these fibres can not be identified in some sections. If the share of such fibres is less than $5 \%$ (see Table 3 ) the analysis can continue, neglecting the non-classified fibres. However, if their share is high, it is possible to identify these fibres through the series and make manual corrections. A high percentage of non-classified fibres also indicates that one or more staining protocols were not optimally adapted to that muscle tissue. In the case of application of antibodies, a high number of the non-classified fibres points either to improper dilution of antibodies or to the lack of antibody specificity. The problematic staining procedures can be repeated and the analysis continued on additional sections whilst the fibre outlines and points defined in the first series of fibre registration can be applied with these additional sections only. If it is necessary to cut new sections it is very often difficult to recognize identical fibres, therefore more than 48 points can be needed to register the new section with the first one.

After the analysis is completed, the above described software is very helpful in recognition of fibres of a chosen type through the whole set of analyzed images. The morphometric data and the optical density (grey level) of every staining are available for each individual fibre as well. Further, after the fibres have been classified through a chosen set of stainings (e.g., immunoperoxidase detection of MyHC isoforms) it is possible to add a new set of reactions (Table 2 and 3 ) to the set of fibres with already identified profile like, e.g., the histochemical reactions to the indicators of oxidative or glycolytic metabolic enzymes or other types of staining that can reveal possible pathological changes in muscle samples.

When combining the fibre classification based on the expression of $\mathrm{MyHC}$ isoforms with the activity of enzymes of energy supplying metabolism, it is very easy to recognize oxidative, glycolytic and oxidative-glycolytic fibres. Further, the software provides also semi-quantitative data on the content of different substances within defined muscle fibres. In this regard the described system can be helpful, e.g., with defining trends in muscle fibre type transformations. The classification is based on thresholding different staining patterns, thus providing data on grey levels for the categories defined by the operator. For instance, in case of analysing the expression of $\mathrm{MyHC}$ isoforms, hybrid fibres expressing two or more isoforms can be easily recognized. Moreover, it is possible to define the prevalence of one or the other isoform within individual fibres based on the profiles to which particular fibres are assigned, e.g., in profile 012 the first component prevails over the second one, while in the profile 102 the second component prevails over the first one. As a conse- 
quence, an insight into the dynamics of muscle fibre type transformation can be studied.

Correlating $\mathrm{MyHC}$ isoform and transcripts expression within a fibre, the so-called "mismatched fibres" could be revealed in muscle samples. These are fibres whose staining profile (combination of dark-0, intermediate- 1 and light-2 fibres) of MyHC isoform expression does not correspond to the MyHC transcripts expression (see Table 3 and Figure 2). The mismatched fibres are the result of a dynamic nature of muscle fibres and are assumed as indicators of fibre type transformations triggered by changed workloads imposed to the muscle (Andersen and Schiaffino, 1997; Talmadge, 2000; Smerdu and Eržen, 2001). For example, fibre No. 35 in Figure 2 was classified as type 1 (stained by BA-D5 antibody), but the expression of corresponding $\beta$-slow MyHC transcripts was very weak or almost absent. This indicates that the transcription of the corresponding MyHC gene had been downregulated due to the fibre transformation and that the transcription of the "new" MyHC gene has not started yet or was too weak to be detected. Such, so-called negative type 1 fibres had been already found in our previous study in human skeletal muscles (Smerdu and Eržen, 2001). Furthermore, since the switch between MyHC isoform expression must be preceded by the switch of MyHC gene expression, the direction of fibre transformation can be determined (Andersen and Schiaffino, 1997; Smerdu and Eržen, 2001). For instance, fibre No. 48 in Figure 2 co-expressed MyHC-1 and $-2 \mathrm{a}$ isoform (1/2a fibre), but only 2a MyHC transcripts, implying that it was transforming probably from type 1 , through type $1 / 2$ a towards type 2 a fibre.

To conclude, the classification procedure described in this paper was inspired by the computer-aided system for the muscle fibre type classification and quantitative analysis developed by Pernuš and co-workers (1986). It is a multipurpose system that can be applied in skeletal muscle research whenever the information about the muscle fibre type pattern is needed. It enables a quick and precise analysis and it is especially helpful when a large number of fibres is analyzed through a large series of differently stained sections within a sample. The system is open, user friendly and very flexible.

\section{Acknowledgements}

This paper was financially supported by the Slovenian Research Agency and the Ministry of Education, Youth and Sports of the Czech Republic (KONTAKT grant No. MEB090910 and grant No. LC06063). The authors acknowledge the skilful technical assistance of Mr. Marko Slak, Mrs. Majda Črnak-Maasarani and Mrs. Andreja Omahen.

\section{References}

Andersen LJ. Schiaffino S. Mismatch between myosin heavy chain $\mathrm{mRNA}$ and protein distribution in human skeletal muscle fibres. Am J Physiol 1997;272:C1881-9.

Bookstein FL. Principal warps: thin-plate splines and the decomposition of deformations. IEEE transaction on pattern analysis and machine intelligence 1989;11:567-85.

Brooke $\mathrm{MH}$, Kaiser KK. Muscle fibre types: how many and what kind? Arch Neurol 1970;23:369-79.

Brox T, Kim Y-J, Weickert J, Feiden W. Fully automated analysis of muscle fiber images with combined region and edge based active contours. In: Proc Bildverarbeitung für die Medizin. Handels $H$, Ehrhardt J, Horsch A, Meinzer HP, Tolxdorff, eds. Springer Verlag, Berlin, 2006, pp. 86-90.

Buche $P$, Mauron D. Quantitative characterization of muscle fiber by image analysis. Computers and Electronics in Agriculture 1997; 16:189-217.

Čapek M, Bruža $P$, Janáček J, Karen $P$, Kubínová L, Vágnerová R. Volume reconstruction of large tissue specimens from serial physical sections using confocal microscopy and correction of cutting deformations by elastic registration. Microsc Res Tech 2009;72:110-9.

Čebašek V, Pernuš F, Obreza S, Ambrož M, Eržen I. Energy metabolism of fibre types within fascicles of human muscles. Pflügers Arch - Eur J Physiol 1996;431:R211-2.

Dahmane R, Valenčič V, Knez N, Eržen I. Evaluation of the ability to make non-invasive estimation of muscle contractile properties on the basis of the muscle belly response. Med Biol Eng Comput 2001;38:51-5.

Dryden IL, Farnoosh R, Taylor CC. Image segmentation using Voronoi polygons and MCMC, with application to muscle fibre images. J Appl Statistics 2006;33:609-22.

Eržen I, Cvetko E, Obreza S, Angaut-Petit D. Fibre types in the mouse levator auris longus muscle: A convenient preparation to study muscle and nerve plasticity. J Neurosci Res 2000;59:692-7.

Eržen I, Primc M, Cvetko E, Sketelj J, D'albis A. Myosin heavy chain profiles in regenerated fast and slow muscles innervated by the same motor nerve become nearly identical. Histochem J 1999;31:27783.

Henckel P, Ducro B, Oksbjerg N, Hassing L. Objectivity of two methods of differentiating fibre types and repeatability of measurements by application of the TEMA image analysing system. Eur J Histochem 1998;42:49-62.

Horton MJ, Brandon CA, Morris TJ, Braun TW, Yaw KM, Sciote JJ. Abundant expression of myosin heavy-chain IIB RNA in a subset of human masseter muscle fibres. Arch Oral Biol 2001; 57:1039-50.

Klemenčič A, Kovačič S, Pernuš F. Automated segmentation of muscle fibre images using active contour models. Cytometry 1998;32:31726.

Kugler P. Microphotometric determination of enzymes in brain sections. Histochemistry 1991;95:579-83.

Lefaucher L, Buche P, Ecolan P, Lemoing M. Classification of pig myofibers and assessment of postmortem glycogen depletion according to fibre type by computerized image analysis. Meat Sci $1992 ; 32: 267-78$.

Likar B, Pernuš F. Registration of serial transverse sections of muscle 
fibers. Cytometry 1999; 37:93-106.

Lucas CA, Kang LHD, Hoh JFY. Monospecific antibodies against the three mammalian fast limb myosin heavy chains. Biochem Biophys Res Commun 2000; 272:303-8.

Meznarič-Petruša M, Eržen I, Zidar J. Dystrophin-significance for diagnostics of neuromuscular diseases. Basic Appl Myol 1992; 2:255-60.

Novikoff AB, Shin WY, Drucker J. Mitochondrial localization of oxidative enzymes: staining results with two tetrasolium salts. J Biochem Cytol 1961;9:47-62.

Otsu NA. Threshold selection method from grey-level histograms. IEEE Trans Sys Man Cyber 1979;9:62-6.

Pernuš F, Bjelogrlič Z, Eržen I. A computer-aided method for muscle fibre type quantification. Acta Stereol 1986;5:49-54.

Pernuš F, Eržen I. Arrangement of fibre types within fascicles of human vastus lateralis muscle. Muscle Nerve 1991;14:304-9.

Pernuš F, Eržen I. Fibre size atrophy and hypertrophy factors in vastus lateralis muscle from 18- to 29-year-old men. J Neurol Sci 1994;121:194-202.

Reichmann H, Pette D. Glycerophosphate oxidase and succinate dehydrogenase activities in IIA and IIB fibres of mouse and rabbit tibialis anterior muscles. Histochemistry 1991;95:5429-33.

Schiaffino S, Gorza L, Sartore S, Saggin L, Vianello M, Gundersen K, Lømo T. Three myosin heavy chain isoforms in type 2 skeletal muscle fibres. J Muscle Res Cell Motil 1989;10:197-205.

Sezgin M, Sankur B. Survey over image thresholding techniques and quantitative performance evaluation. $J$ Electronic Imaging 2004;13:146-65.

Smerdu V, Karsch Mizrachi I, Campione M, Leinwand L, Schiaffino S. Type IIx myosin heavy chain transcripts are expressed in IIb fibers of human skeletal muscle. Am J Physiol 1994;267:C1723-B
Smerdu V, Eržen I. Dynamic nature of fibre-type specific expression of myosin heavy chain transcripts in 14 different human skeletal muscles. J Muscle Res Cell Motil 2001;22:647-55.

Smerdu V, Soukup T. Demonstration of myosin heavy chain isoforms in rat and humans: the specificity of seven available monoclonal antibodies used in immunohistochemical and immunoblotting methods. Eur J Histochem 2008;52:179-90.

Snoj-Cvetko E, Eržen I. Histochemical and morphometric characteristics of the vastus lateralis muscle in children. Basic Appl Myol 1992;2:277-84.

Snoj-Cvetko E, Sketelj J, Dolenc I, Obreza S, Janmot C, D'albis A, Eržen I. Regenerated rat fast muscle transplanted to the slow muscle bed and innervated by the slow nerve exhibits and identical myosin heavy chain repertoire to that of the slow muscle. Histochemistry 1996a;106:473-9.

Snoj-Cvetko E, Smerdu V, Sketelj J, Dolenc I, D'albis A, Janmot C, Eržen I. Adaptive range of myosin heavy chain expression in regenerating soleus is broader than in mature muscle. J Muscle Res Cell Motil 1996b;17:401-9

Škorjanc D, Šalehar A, Eržen I, Kastelic M. Pattern of fibre type distribution within muscle fascicles of pigs (Sus scrofa domestica). Czech J Anim Sci 2007;52:89-95.

Talmadge RL. Myosin heavy chain isoform expression following reduced neuromuscular activity: potential regulatory mechanisms. Muscle Nerve 2000;23:661-79.

Travnik L, Pernuš F, Eržen I. Histochemical and morphometric characteristics of the normal human vastus medialis longus and vastus medialis obliquus muscles. J Anat 1995;187:403-11.

Weiss A, McDonough D, Wertman B, Acakpo-Satchivi L, Montgomery K, Kucherlapati R, Leinwand LA, Krauter K. Organization of human and mouse skeletal myosin heavy chain gene clusters is highly conserved. Proc Natl Acad Sci USA 1999;96:2958-63. 